\title{
Two carrier temperatures non-equilibrium generalized Planck law for semiconductors
}

\author{
François Gibelli $^{\mathrm{a}, \mathrm{b}, *}$, Laurent Lombez ${ }^{\mathrm{a}, \mathrm{b}, \mathrm{c}}$, Jean-François Guillemoles ${ }^{\mathrm{a}, \mathrm{b}, \mathrm{c}}$ \\ a Institute of Research and Development of Photovoltaic Energy IRDEP (CNRS-ENSCP-EDF), UMR 7174, 6 quai Watier, 78401 Chatou, France \\ ${ }^{\mathrm{b}}$ NextPV, International Associate Laboratory (LIA), 4-6-1 Komaba, Meguro-ku, Tokyo 153-8904, Japan \\ ${ }^{c}$ Ile-de-France Photovoltaic Institute (IPVF), 8, rue de la Renaissance, 92160 Antony, France
}

\section{A R T I C L E I N F O}

Article history:

Received 20 January 2016

Received in revised form

6 June 2016

Accepted 8 June 2016

Available online 16 June 2016

Keywords:

Non-equilibrium carriers

Generalized Planck's law

Absolute electrochemical potentials

Emission temperature

Photoluminescence

Hot carrier

\begin{abstract}
A B S T R A C T
Planck's law of radiation describes the light emitted by a blackbody. This law has been generalized in the past for the case of a non-blackbody material having a quasi Fermi-level splitting: the lattice of the material and the carriers are then considered in an isothermal regime. Hot carrier spectroscopy deals with carriers out of the isothermal regime, as their respective temperatures $\left(T_{H}^{e} \neq T_{H}^{h}\right)$ are considered to be different than that of the lattice $\left(T_{L}\right)$. Here we show that Fermi-Dirac distribution temperature for each type of carrier still determine an effective radiation temperature: an explicit relationship is given involving the effective masses. Moreover, we show how to determine, in principle with an additional approximation, the carrier temperatures $\left(T_{H}^{e}, T_{H}^{h}\right)$ and the corresponding absolute electrochemical potentials from photoluminescence measurements.
\end{abstract}

(c) 2016 Elsevier B.V. All rights reserved.

\section{Introduction}

The spectral distribution of light emitted by any piece of matter brings a wealth of information. At thermal equilibrium, it is described by Planck's radiation law [1] and it informs on the absorptivity (hence the electronic structure of the material) and temperature. This law has been generalized by Lasher and Stern [2], De Vos and Pauwels [3] and Würfel [4] for materials in quasiequilibrium. This so called "generalized Planck's law" describes the emitted photon flux from electron and hole populations, where carriers can still be described by a Fermi Dirac distribution (i.e. temperature and chemical potentials can be defined) and which are at the same temperature. This equation has been applied with success to determine absorption [5-7], quasi-Fermi level splitting $[8,9]$ or temperature [10-12] with direct applications in photovoltaic research [13-15].

This law is currently being used to measure the quasi-Fermi level separation in excited semiconductors and has therefore a considerable potential to be used in, e.g., investigations on photovoltaic effect by luminescence analysis [9,16-18]. Photoluminescence experiments are often carried out under high

\footnotetext{
* Corresponding author at: Institute of Research and Development of Photovoltaic Energy IRDEP (CNRS-ENSCP-EDF), UMR 7174, 6 quai Watier, 78401 Chatou, France.

E-mail address: francois.gibelli@etu.upmc.fr (F. Gibelli).
}

excitation, conditions where thermal equilibrium of carriers with the lattice is not always valid, and, because they relax at different rates, the thermal equilibrium between the electrons subsystem and the holes subsystem is not always valid either. Such situations are commonly encountered in recent scientific studies (transistors, diodes, hot carrier solar cells, etc.) [19-23] including hot topics such as carrier relaxation in 2D materials such as graphene [2427] and dichaocogenides [28].

In order to investigate the properties of hot carriers in a wide range of different semiconducting devices, luminescence analysis has been widely used to estimate the energy distribution of the carrier population [10,12,29-35].

In luminescence experiments, a light beam generates electronhole pairs in the material and the light emitted by the sample by carrier recombination is recorded as a photoluminescence spectrum. Assuming a constant temperature and electrochemical potential in the probed region of the sample, this photoluminescence spectrum obeys the generalized Planck's law [2,4]:

$I_{P L}(\hbar \omega)=A(\hbar \omega) \frac{\Omega n_{o p}^{2}(\hbar \omega)^{2}}{4 n^{2} \hbar^{3} c_{0}^{2}}\left[\exp \left(\frac{\hbar \omega-\Delta \mu}{k_{B} T}\right)-1\right]^{-1}$

where $\hbar \omega$ is the energy of the emitted photon, $A(\hbar \omega)$ the energy and excitation [13] dependant absorption of the semiconductor, $\Omega$ the solid angle for emission, $n_{o p}$ the optical index of the semiconductor, $c_{0}$ the velocity of the light in vacuum, $k_{B}$ the Boltzmann 
constant, $T$ the temperature of the semiconductor and $\Delta \mu$ the quasi-Fermi level splitting written as an electrochemical potential difference.

From the photoluminescence spectrum, a spectral shift indicates a change in the lattice temperature, the intensity is related to the quasi-Fermi level splitting and the slope at energies close but higher than that of the peak shows the emission temperature variation. Indeed, Planck indicates [1] that the temperature included in his radiation law is an emission temperature, defined as that of a body in equilibrium with the radiation.

The temperature of the carrier is a true challenge for the community, theoretically and experimentally. While some consider that a same temperature describes the electrons and the holes that are in thermal equilibrium [21,36-39], others think that the holes, having a higher effective mass, are always close to the lattice temperature [40] and that only electrons are hot, or that the majority carriers are fully thermalized whereas only the minority ones are hot [21]. Apart from the value of these temperatures, it remains a hard task to know the meaning of the temperature in the generalized Planck's law in non-equilibrium conditions, and especially to determine which carrier is mostly affected by hot carrier effects.

Here we propose a more complete analysis, where the radiation law is written with electron and hole populations which are not at the same temperature.

Indeed we show the expression of the generalized Planck's law for different electron and hole temperatures. This law enables us to verify the different assumptions made about the nature of the individual hot carrier population and offers new perspectives in advanced optical characterization of materials. The effective mass ratio appears to be the critical parameter that relates the radiation temperature to the two carrier temperatures. The usefulness of the developed expression is discussed for the case of hot carrier solar cells since the knowledge of the two carrier temperatures is a key point towards the development of energy selective contacts.

\section{Theory}

\subsection{General expression}

The transition from Planck's law to its generalized expression has been done by Lasher and Stern [2] and later by de Vos and Pauwels [3] and Würfel [4]: their method roots on the balance between the absorption, spontaneous and stimulated emission of the semiconductor. These general expressions depend only on the transition matrix element and on the Fermi-Dirac distribution of carriers. In the presence of hot carriers, as the lattice remains constant and close to ambient temperature, the transition matrix elements are the same as in the absence of hot carriers, as the density of states is the same. Using very general expressions of Fermi-Dirac distributions, namely $f_{V}^{e}, f_{C}^{e}$ as the distributions of the electrons in the valence and in the conduction band respectively, Würfel [41] gives the expression of the photoluminescence intensity:

$I_{P L}(\hbar \omega)=A(\hbar \omega) \frac{\Omega n_{o p}^{2}(\hbar \omega)^{2}}{4 \pi^{3} \hbar^{3} c_{0}^{2}} \frac{\left[1-f_{V}^{e}\left(E^{h}\right) f_{C}^{e}\left(E^{e}\right)\right.}{f_{V}^{e}\left(E^{h}\right)-f_{C}^{e}\left(E^{e}\right)}$,

where the general distributions for electron and for holes are respectively:

$f_{V}^{e}\left(E^{h}\right)=\left[1+\exp \left(\frac{E^{h}-E_{F_{p}, H}}{k_{B} T_{H}^{h}}\right)\right]^{-1} f_{C}^{e}\left(E^{e}\right)=\left[1+\exp \left(\frac{E^{e}-E_{F_{n}, H}}{k_{B} T_{H}^{e}}\right)\right]^{-1}$ where $E^{e, h}$ is the energy of the electron (hole), $\mu^{e, h}$ the electrochemical potential and $T^{e, h}$ the temperature.

Using these distributions leads to the general expression of Planck's law with two temperatures:

$I_{P L}(\hbar \omega)=A(\hbar \omega) \frac{\Omega n^{2}(\hbar \omega)^{2}}{4 n^{2} \hbar^{3} c^{2}} \times\left[\exp \left(\frac{E^{e}(\hbar \omega)-E_{F_{n}, H}}{k_{B} T_{H}^{e}}-\frac{E^{h}(\hbar \omega)-E_{F p, H}}{k_{B} T_{H}^{h}}\right)-1\right]^{-1}$

where $A(\hbar \omega)$ is considered to be the same spectral absorptivity than that appearing in Planck's law, as can be inferred by setting $T_{H}^{e}=T_{H}^{h}=T, E_{F n}=E_{F p}$ and $E^{e}-E^{h}=\hbar \omega$.

If $T_{H}^{e}=T_{H}^{h}=T$ and $E^{e}-E^{h}=\hbar \omega$, expression (1) is recovered. In expression (2) because of the different temperatures, the energy of the electron $E^{e}$ and hole $E^{h}$ have to be expressed explicitly.

Rather than using quasi-Fermi levels, the energies of the carriers will be expressed relatively to their electrochemical potential $\mu_{H}^{e}, \mu_{H}^{h}$ [41]. In order to express the energies of the electron $E^{e}$ and hole $E^{h}$, a known energy reference has to be determined. This reference is classically the vacuum energy; for the semiconducting materials considered here, the middle of the energy gap seems to be much more appropriate. The different energies are shown relative to this reference in Fig. 1.

Since an energy reference has been defined, it is possible to determine the energy of the electrons and holes for different material structures, and therefore write a generalized Planck's law for non-equilibrium carrier for semiconductor in permanent regime [42].

In this work, we suppose that the bands are isotropic and parabolic and the energy reference is taken at the mid-gap around $\Gamma$ in the Brillouin zone. So the energy of the conduction and valence band edges are $E_{c}=E_{G} / 2$ and $E_{v}=-E_{G} / 2$ respectively.

\subsection{Generalized Planck's law expressions}

The dispersion relation of a bulk semiconductor gives the energy of the electron in the conduction band $E^{e}$ and that of the hole in the valence band $E^{h}$, with $m^{e}, m^{h}$ the effective mass of the electron in the conduction band and of the hole in the valence band respectively, $\mathbf{k}$ the wavevector for the considered radiative transition:

$E^{e}=\frac{E_{G}}{2}+\frac{\hbar^{2} \mathbf{k}^{2}}{2 m^{e}} E^{h}=-\frac{E_{G}}{2}-\frac{\hbar^{2} \mathbf{k}^{2}}{2 m^{h}}$

$\mathrm{p}$ region $\quad \mathrm{n}$ region $E_{\text {vacuum }}$

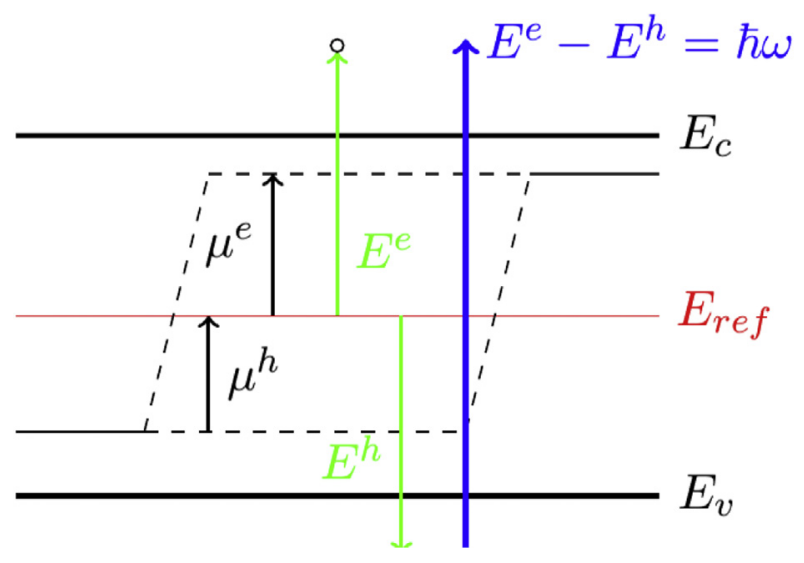

Fig. 1. Energy reference used in this work. 
knowing that $E^{e}-E^{h}=\hbar \omega$ :

$\mathbf{k}^{2}=\frac{2}{\hbar^{2}}\left(\hbar \omega-E_{G}\right)\left(\frac{m^{e} m^{h}}{m^{e}+m^{h}}\right)$

substituting $\mathbf{k}$ in the electron and hole energies above gives the joint states:

$E^{e}=\frac{E_{G}}{2}+\left(\hbar \omega-E_{G}\right) \frac{m^{h}}{m^{e}+m^{h}} E^{h}=-\frac{E_{G}}{2}-\left(\hbar \omega-E_{G}\right) \frac{m^{e}}{m^{e}+m^{h}}$

introducing these last expressions in expression (2) the emitted photon flux of a bulk material in the presence of hot carriers writes

$$
\begin{aligned}
I_{P L, B}(\hbar \omega)= & A(\hbar \omega) \\
& \times \frac{\Omega n^{2}(\hbar \omega)^{2}}{4 \pi^{2} \hbar^{3} c_{0}^{2}} \\
& \times\left[\operatorname { e x p } \left(\frac{E_{G}}{2 k_{B}}\left(\frac{m^{e}-m^{h}}{m^{e}+m^{h}}\right)\left(\frac{1}{T_{H}^{e}}-\frac{1}{T_{H}^{h}}\right)\right.\right. \\
& \left.\left.+\frac{\hbar \omega}{k_{B}\left(m^{e}+m^{h}\right)}\left(\frac{m^{h}}{T_{H}^{e}}+\frac{m^{e}}{T_{H}^{h}}\right)-\frac{\mu_{H}^{e}}{k_{B} T_{H}^{e}}-\frac{\mu_{H}^{h}}{k_{B} T_{H}^{h}}\right)-1\right]^{-1}
\end{aligned}
$$

This law describes the luminescence of semiconducting material out of equilibrium with electron and hole reservoirs with different thermodynamic fundamental quantities.

Similar expressions can be found when considering bulk material confined in 1 or 2 dimensions. For the quantum well structure (see Appendix A), the generalized Planck's law is given by the following expression:

$$
\begin{aligned}
I_{P L, Q W e}(\hbar \omega)= & C(\hbar \omega) \\
\times & {\left[\operatorname { e x p } \left(\frac { 1 } { k _ { B } } ( \frac { 1 } { T _ { H } ^ { e } } - \frac { 1 } { T _ { H } ^ { h } } ) \left[\left(\frac{m^{e}}{m^{e}+m^{h}}\right)\left(\frac{E_{G}}{2}+E_{c}^{i}\right)\right.\right.\right.} \\
& \left.-\left(\frac{m^{h}}{m^{e}+m^{h}}\right)\left(\frac{E_{G}}{2}+\mid E_{v}^{j^{\prime}}\right)\right] \\
& \left.\left.+\frac{\hbar \omega}{k_{B}\left(m^{e}+m^{h}\right)}\left(\frac{m^{h}}{T_{H}^{e}}+\frac{m^{e}}{T_{H}^{h}}\right)-\frac{\mu_{H}^{e}}{k_{B} T_{H}^{e}}-\frac{\mu_{H}^{h}}{k_{B} T_{H}^{h}}\right)-1\right]^{-1}
\end{aligned}
$$

In a quantum wire the generalized Planck's law writes (see Appendix A):

$$
\begin{aligned}
I_{P L, Q W i}(\hbar \omega)= & C(\hbar \omega) \\
& {\left[\operatorname { e x p } \left(\frac { 1 } { k _ { B } } ( \frac { 1 } { T _ { H } ^ { e } } - \frac { 1 } { T _ { H } ^ { h } } ) \left[\left(\frac{m^{e}}{m^{e}+m^{h}}\right)\left(\frac{E_{G}}{2}+E_{c, y}^{i}+E_{c, z}^{i}\right)\right.\right.\right.} \\
& \left.-\left(\frac{m^{h}}{m^{e}+m^{h}}\right)\left(\frac{E_{G}}{2}+\left|E_{v, y}^{j}\right|+\mid E_{v, z}^{j}\right)\right] \\
& \left.\left.+\frac{\hbar \omega}{k_{B}\left(m^{e}+m^{h}\right)}\left(\frac{m^{h}}{T_{H}^{e}}+\frac{m^{e}}{T_{H}^{h}}\right)-\frac{\mu_{H}^{e}}{k_{B} T_{H}^{e}}-\frac{\mu_{H}^{h}}{k_{B} T_{H}^{h}}\right)-1\right]^{-1}
\end{aligned}
$$

where

$C(\hbar \omega)=A(\hbar \omega) \frac{\Omega n_{o p}^{2}(\hbar \omega)^{2}}{4 \pi^{2} \hbar^{3} c^{2}}$

Applying the method presented in Appendix A will lead to a general form of expression (2):

$$
\begin{aligned}
& I_{P L}(\hbar \omega)=C(\hbar \omega) \\
& {\left[\exp \left(\varepsilon\left(T_{H}^{e}, T_{H}^{h}\right)+\frac{\hbar \omega}{k_{B}\left(m^{h}+m^{e}\right)}\left(\frac{m^{h}}{T_{H}^{e}}+\frac{m^{e}}{T_{H}^{h}}\right)-\frac{\mu_{H}^{e}}{k_{B} T_{H}^{e}}-\frac{\mu_{H}^{h}}{k_{B} T_{H}^{h}}\right)-1\right]^{-1}}
\end{aligned}
$$

where $C$ was defined previously in expression (6), and $\varepsilon$ a function of the hot temperatures of electrons and holes and depending on known parameters (effective masses, constants, confinement energies, etc.).

By replacing $T_{H}^{e}$ and $T_{H}^{h}$ with $T$ in all the above given expressions of the generalized Planck's law with two temperatures (expressions (2)-(5)), the new expression obtained is the generalized Planck's law of expression (1). The developed formalism is thus in accordance with the classical results.

Comparing the generalized Planck's law with two different temperatures (2) with the generalized Planck's law of expression (1) shows the relationship between the effective temperature $T_{H}$, the effective quasi-Fermi level splitting $\Delta \mu_{H}$, and the absolute parameters $T_{H}^{e}, T_{H}^{h}, \mu_{H}^{e}, \mu_{H}^{h}$ :

$T_{H}=\frac{\left(m^{e}+m^{h}\right) T_{H}^{e} T_{H}^{h}}{m^{h} T_{H}^{h}+m^{e} T_{H}^{e}}$

$\Delta \mu_{H}=\frac{\left(m^{e}+m^{h}\right) T_{H}^{e} T_{H}^{h}}{m^{h} T_{H}^{h}+m^{e} T_{H}^{e}}\left(\frac{\mu_{H}^{e}}{T_{H}^{e}}+\frac{\mu_{H}^{h}}{T_{H}^{e}}-\varepsilon\left(T_{H}^{e}, T_{H}^{h}\right)\right)$

\section{Results and discussion}

\subsection{Effective masses ratio and carrier temperatures}

We now draw some general discussions that can be obtained even when the temperature of each carrier type is not known.

The emission temperature as a function of the carrier temperatures can be written as (see Appendix B for details):

$1=\alpha \frac{T_{H}}{T_{H}^{h}}+(1-\alpha) \frac{T_{H}}{T_{H}^{e}}$

introducing $x=T_{H} / T_{H}^{h}, y=T_{H} / T_{H}^{e}$, we obtain

$y=\frac{1-\alpha x}{1-\alpha}$

with

$\alpha=\frac{m^{e}}{m^{e}+m^{h}}$

$\left.\left(m^{e}, m^{h}\right) \in \mathbb{R}^{+*} \times \mathbb{R}^{+*} \Longrightarrow \alpha \in\right] 0,1[$

This relationship is illustrated in Fig. 2, where we plot the ratio $\frac{T_{H}}{T_{H}^{e}}$ for different couples of $\left(\alpha, \frac{T_{H}}{T_{H}^{h}}\right)$.

In order to study which carrier has a temperature close to the emission temperature according to the value of the effective mass ratio $\alpha$, we will consider here $(x, y) \in[0.5,2] \times[0.5,2]$. This can be safely assumed at least when the excitation power is not too large. Indeed, under low injection $T_{H}^{e}=T_{H}^{h}=T_{L}$. Increasing the excitation saturates the relaxation channels leading to a partial decoupling of the temperatures. In these regions three different cases can be identified:

- If $\alpha \in] 0,0.05]$ and $x$ is allowed to vary, $y \in[0.95,1.03]$, that is, the emission temperature is mainly due to the hot temperature of the electrons as the variation between the emission temperature and that of the electrons is between $0.03 \%$ and $0.05 \%$. 


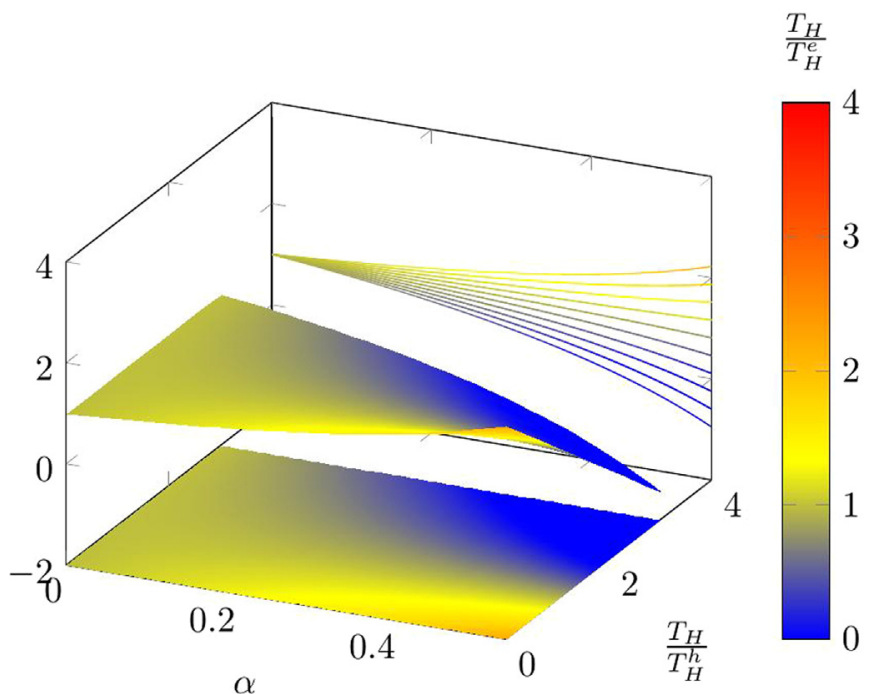

Fig. 2. Carrier and emission temperature variations with the effective mass related quantity $\alpha$, expression (11). The colormap at the bottom is the projected surface plot, showing the variation of carrier and emission temperatures according to the legend of the colorbar. The line plot is the projection of the surface plot, showing the variation of $\frac{T_{H}}{T \rho}$ with $\alpha$. (For interpretation of the references to color in this figure caption, the ${ }^{T}$ reader is referred to the web version of this paper.)

This is shown on the projected line plot of Fig. 2: for $\alpha$ close to zero, all line plots converge to 1 , indicating $T_{H}^{e} \simeq T_{H}$.

- If $\alpha \in[0.95,1[$ and $y$ is allowed to vary, $x \in[0.95,1.03]$ and the emission temperature is mainly due to the hot temperature of the holes; this case is symmetric to the first one listed here.

- If $\alpha=0.5$, then $y=2-x$ : equal effective masses does not imply that carriers are at the same temperature, as shown on the projected colormap in Fig. 2: at $\alpha=0.5$, the variation in $\frac{T_{H}}{T_{H}^{h}}$ induced a change in $\frac{T_{H}}{T_{H}^{e}}$, indicating that the carriers do not have the same temperature.

For all other effective mass ratio it is not possible to conclude about a clear relationship between one type of carrier temperature and the emission temperature.

This can be shown in an alternative way, by rewriting expression (8) as:

$$
\frac{T_{H}^{e}}{T_{H}^{h}}=\frac{\frac{T_{H}^{e}}{T_{H}}+1}{\alpha}-1
$$

giving the ratio of the carrier temperatures versus the effective mass ratio and the ratio between the temperature of the electrons and the emission temperature. This expression confirms the properties of the three domains indicated above as it is shown in Fig. 3.

Now if we look at real cases of materials listed in Table 1, only InAs can be considered containing hot electrons and cold holes because $\alpha$ is very close to 0.05 . For the two other materials, reporting the value of $\alpha$ in Fig. 2, the electrons have probably a higher temperature than the emission temperature, and the holes are also heated, but at a lower temperature than the emission temperature $\left(T_{L}<T_{H}^{h}<T_{H}<T_{H}^{e}\right)$.

For the materials developed for hot carrier solar cell applications [43,39], the value of $\alpha$ is typically between 0.01 and 0.5 ; as electrons have a smaller effective mass than the holes, they get a higher share of the energy of the incident photons. If the cooling of electrons is slower or identical to that of holes, electrons then have a temperature above the emission temperature, whereas the holes are colder. As it turns out, this is the case in hot phonon bottleneck where cooling rates increase with the effective mass [44].

Because we describe situations where energy is brought to the electronic system, we have the following relationships, where $T_{L}$ is the temperature of the lattice:

$T_{L} \leq \min \left(T_{H}, T_{H}^{e}, T_{H}^{h}\right)$

$\min \left(T_{H}^{e}, T_{H}^{h}\right) \leq T_{H} \leq \max \left(T_{H}^{e}, T_{H}^{h}\right)$

$T_{H}^{e} \leq \frac{2 m^{h}\left[\langle\hbar \omega\rangle-E_{G}\right]}{3 k_{B}\left(m^{e}+m^{h}\right)}$

$T_{H}^{h} \leq \frac{2 m^{e}\left[\langle\hbar \omega\rangle-E_{G}\right]}{3 k_{B}\left(m^{e}+m^{h}\right)}$

where $\langle\hbar \omega\rangle$ is the average energy of the incident photons per photon. Moreover, unless Auger processes are particularly effective, the temperature $\max \left(T_{H}^{e}, T_{H}^{h}\right)$ is usually bounded by the excess energy given to the electron-hole pairs $\left(\hbar \omega-E_{G}\right.$ in the case of photoluminescence); even if one carrier gets all, there is a limit: $3 / 2 k_{B} T_{H}^{e, h} \leq\left(\langle\hbar \omega\rangle-E_{G}\right) \frac{m^{h, e}}{m^{e}+m^{h}}$.

These temperature relative values can be studied qualitatively as functions of the absorbed light power, since at low incident photon flux the thermalization is rapid whereas at high incident photon flux the thermalization slows down due to the phonon bottleneck effect. Indeed many different experimental works have shown that the observed emission temperature $T_{H}$ grows with the absorbed power [29,12,32], as shown with the black bullets in Fig. 4.

Following up with the discussion above, two limiting cases can be considered: (1) $T_{H}^{e}=T_{H}^{h}=T_{H}$ and (2) $T_{H}^{h} \simeq T_{L}$, one carrier gets virtually all excess of energy. These cases are depicted in Fig. 4, by respectively the thick line for case (1) and the dashed line electrons and the dotted line for holes in case (2). Between these two extreme cases, $T_{H}^{e}=T_{H}^{h}=T_{H}$ and $T_{H}^{h}=T_{L}=300 \mathrm{~K}$, all combinations verifying $T_{H}^{e} \geq T_{H}$ and $T_{H}^{h} \leq T_{H}$ are possible. For example we have shown one of these possibilities in Fig. 4 with the squares standing for the hole temperature and stars for the electron temperature.

In Fig. 4, if we consider that the isothermal emission temperature follows the thick line whereas the electron and hole temperatures are represented by the stars and the squares respectively then we can identify two regions of interest:

A. In region A the temperature of the holes is very close to the lattice, $T_{H}^{h} \simeq T_{L}$. With this assumption, expression (8) can be rewritten as:

$T_{H, \text { max }}^{e}=T_{H}\left(\frac{1}{1-\frac{\alpha}{1-\alpha} \frac{T_{H}-T_{L}}{T_{L}}}\right)$

Region A starts from low power where $T_{H}^{e}=T_{H}^{h}=T_{H}=T_{L}$ to the point where $\frac{\alpha}{1-\alpha} \frac{T_{H}-T_{L}}{T_{L}}<\frac{T_{H}-T_{L}}{T_{L}} \ll 1$ : the temperature of electrons can be considered to be the same as the emission temperature.

B. In region $\mathrm{B}, T_{H}^{e} \geq T_{H}$ and $T_{C} \leq T_{H}^{h} \leq T_{H}$ : both carriers are significantly heated.

This first theoretical insight into the different electron and hole temperatures and their variation with the absorbed light power enables us to show the different regimes occurring during heating both carrier types. The lightest carriers heat first and carry more heat than the heaviest ones. 


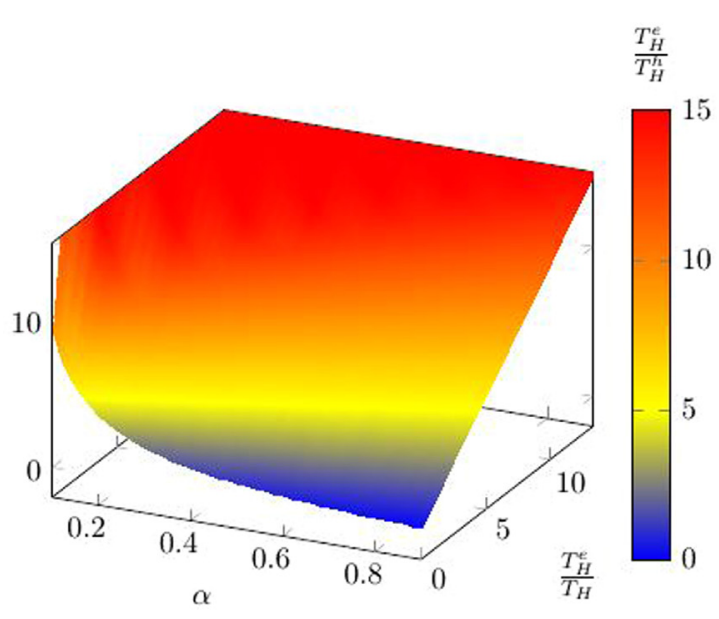

(a) $3 \mathrm{D}$ representation

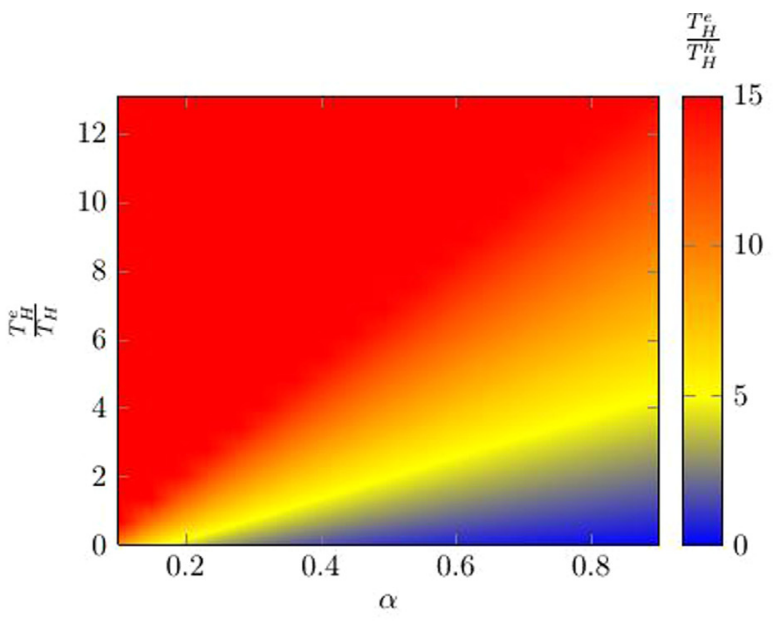

(b)Colormap representation

Fig. 3. Second representation of the relationship between emission temperature and carrier temperatures, expression (14).

Table 1

Effective mass ratio for three classical III-V hot carrier materials.

\begin{tabular}{llll}
\hline Material & $m^{e}\left(\cdot m_{0}\right)$ & $m^{h}\left(\cdot m_{0}\right)$ & $\alpha$ \\
\hline InAs & 0.023 & 0.41 & 0.053 \\
$\mathrm{Ga}_{0.47} \mathrm{In}_{0.53} \mathrm{As}$ & 0.041 & 0.45 & 0.083 \\
$\mathrm{InP}$ & 0.08 & 0.6 & 0.117 \\
\hline
\end{tabular}

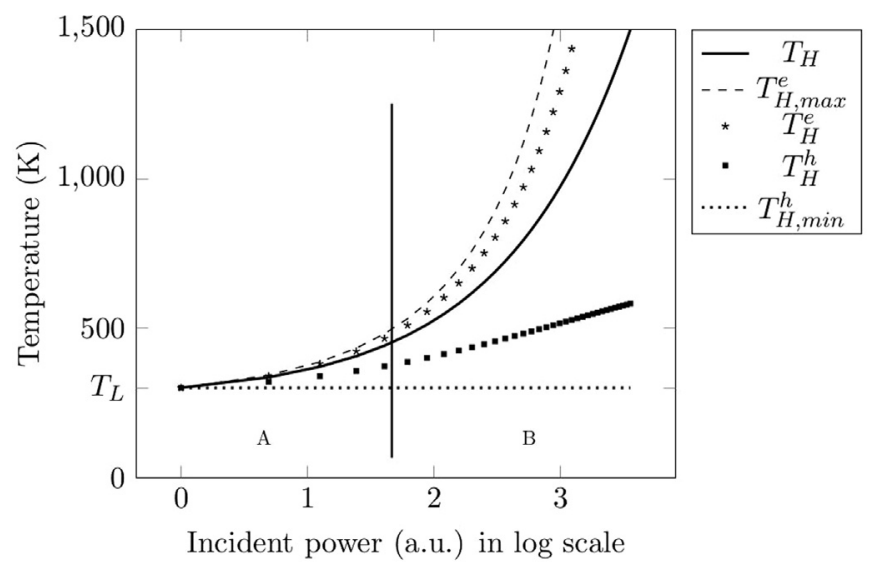

Fig. 4. Variation of the temperatures with the absorbed light power: the thick curve corresponds to the emission temperature $T_{H}^{e}=T_{H}^{h}=T_{H}$ (case 1), the dashed and dotted curves represents the electron and hole temperatures respectively when $T_{H}^{e}=T_{L}=300 \mathrm{~K}$ (case 2 ), and the stars and squares are a possible electron and hole temperature shape of the material (see text). Here $\alpha \leq 0.5$. The vertical line represents the limit where the temperature of electrons can be considered equal to the emission temperature and corresponds to $\frac{\alpha}{1-\alpha} \frac{T_{H}-T_{L}}{T_{L}} \ll 1$. For $\alpha=0.5$, the theoretical maximal achievable carrier temperatures given in expressions (17) and (18) $T_{H, \max }^{e}=T_{H, \max }^{h}=2166 \mathrm{~K}$, in accordance with the displayed results.

\subsection{Determination of absolute electrochemical potentials}

The literature about hot carriers generally make some assumptions not only about the temperature, as explained in the introduction, but also on the electrochemical potential of each carrier type. The most common of them is to consider that electrons and holes have same effective masses and same dynamics, so $\mu_{H}^{e}= \pm \mu_{H}^{h}= \pm \frac{\Delta \mu_{H}}{2}$ are equal (or symmetric, according to the choice of the energy reference), and equal to half of the electrochemical potential difference given in the classical generalized Planck's law.

Starting from the expression (9) giving the relationship between the electrochemical potential difference of the classical generalized Planck's law (expression (1)) and the parameters of each type of carrier, two cases will be studied. Here we consider a material of InGaAsP in which hot carrier have been observed [33]. The model taken here is the bulk generalized Planck's law with two temperatures, so that:

$\Delta \mu_{H}=\frac{\left(m^{e}+m^{h}\right) T_{H}^{e} T_{H}^{h}}{m^{h} T_{H}^{h}+m^{e} T_{H}^{e}}\left(\frac{\mu_{H}^{e}}{T_{H}^{e}}+\frac{\mu_{H}^{h}}{T_{H}^{h}}-\frac{E_{G}}{2}\left(\frac{m^{e}-m^{h}}{m^{e}+m^{h}}\right)\left(\frac{1}{T_{H}^{e}}-\frac{1}{T_{H}^{h}}\right)\right)$

In the experimental conditions, electrons and holes are generated and recombine together, therefore, electroneutrality holds. It writes:

$\left(\frac{m^{e} T_{H}^{e}}{m^{h} T_{H}^{h}}\right)^{3 / 2}=\exp \left[\frac{E_{G}}{2 k_{B}}\left(\frac{1}{T_{H}^{e}}-\frac{1}{T_{H}^{h}}\right)+\frac{\mu_{H}^{h}}{k_{B} T_{H}^{h}}-\frac{\mu_{H}^{e}}{k_{B} T_{H}^{h}}\right]$

Appendix $C$ shows that this electroneutrality can be combined with expression (9) giving $\Delta \mu_{H}$ to have only temperature dependant absolute electrochemical potentials:

$$
\begin{aligned}
\mu_{H}^{h}= & \frac{T_{H}^{h}}{2}\left[\frac{\left(\alpha T_{H}^{e}+(1-\alpha) T_{H}^{h}\right) \Delta \mu_{H}}{T_{H}^{e} T_{H}^{h}}+E_{G}(\alpha-1)\left(\frac{1}{T_{H}^{e}}-\frac{1}{T_{H}^{h}}\right)\right. \\
& \left.+\frac{3}{2} k_{B} \ln \left(\frac{\alpha T_{H}^{e}}{(1-\alpha) T_{H}^{h}}\right)\right]
\end{aligned}
$$

$$
\begin{aligned}
\mu_{H}^{e}= & \frac{T_{H}^{e}}{2}\left[\frac{\left(\alpha T_{H}^{e}+(1-\alpha) T_{H}^{h}\right) \Delta \mu_{H}}{T_{H}^{e} T_{H}^{h}}+\alpha E_{G}\left(\frac{1}{T_{H}^{e}}-\frac{1}{T_{H}^{h}}\right)\right. \\
& \left.-\frac{3}{2} k_{B} \ln \left(\frac{\alpha T_{H}^{e}}{(1-\alpha) T_{H}^{h}}\right)\right]
\end{aligned}
$$

Let us consider first the case of identical electron and hole effective masses, $m^{e}=m^{h}$. Under the assumption $T_{H}=2 T_{H}^{e} T_{H}^{h} /\left(T_{H}^{e}+T_{H}^{h}\right)$, and the expressions (20) and (21) become: 


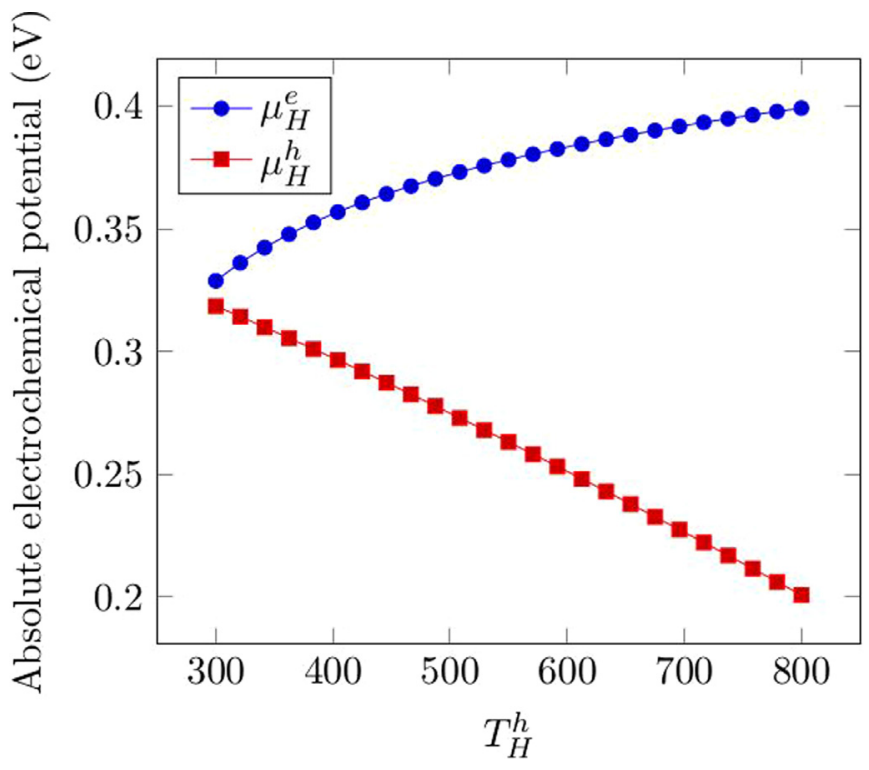

Fig. 5. Variation of electrochemical potentials with the carrier temperature at an emission temperature of $T_{H}=800 \mathrm{~K}$, and $\Delta \mu_{H}=0.6 \mathrm{eV}$ for an InGaAsP alloy, with $m^{e}=0.069 m_{0}, m^{h}=0.47 m_{0}, E_{G}=0.7 \mathrm{eV}$.

$\mu_{H}^{h}=\frac{T_{H}^{h}}{4}\left[\frac{\left(T_{H}^{e}+T_{H}^{h}\right) \Delta \mu_{H}}{T_{H}^{e} T_{H}^{h}}+E_{G}\left(\frac{1}{T_{H}^{e}}-\frac{1}{T_{H}^{h}}\right)+3 k_{B} \ln \left(\frac{T_{H}^{e}}{T_{H}^{h}}\right)\right]$

$\mu_{H}^{e}=\frac{T_{H}^{e}}{4}\left[\frac{\left(T_{H}^{e}+T_{H}^{h}\right) \Delta \mu_{H}}{T_{H}^{e} T_{H}^{h}}+E_{G}\left(\frac{1}{T_{H}^{e}}-\frac{1}{T_{H}^{h}}\right)-3 k_{B} \ln \left(\frac{T_{H}^{e}}{T_{H}^{h}}\right)\right]$

Expressions (22) and (23) are not equal, because $T_{H}^{e} \neq T_{H}^{h}$. If the assumption of a same hot temperature of electrons and holes is added to the assumption of equal effective masses, then $T_{H}^{e}=T_{H}^{h}=T_{H}$ and $\mu_{H}^{e}=\mu_{H}^{h}=\Delta \mu_{H} / 2$. So the classical assumption made about the electrochemical potentials is quite verified only if same effective masses and same temperature of both carriers are considered.

Coming back to expressions (20) and (21), we will consider now different effective masses and different carrier temperatures. From the literature [45] we take $m^{e}=0.069 m_{0}, m^{h}=0.47 m_{0}, E_{G}=0.7 \mathrm{eV}$, corresponding to InGaAsP III-V semiconducting alloy.

Fig. 5 represents the variations of the absolute electrochemical potentials $\mu_{H}^{e}, \mu_{H}^{h}$ as a function of the temperature of the holes $T_{H}^{h}$, for a typical illumination (see Ref. [33]). The absolute electrochemical potentials vary only with one temperature, because that of the other carrier type is given by expression (8). From Fig. 5 it can be inferred that the absolute chemical potentials are very close for $T_{H}^{h}=300 \mathrm{~K}$. For holes hotter than $T_{H}^{h}=300 \mathrm{~K}$, the absolute electrochemical potential of electrons increases to reach $\mu_{H}^{e}=0.4 \mathrm{eV}$ at $T_{H}^{h}=T_{H}=800 \mathrm{~K}$ whereas that of the holes decreases to $\mu_{H}^{h}=0.2 \mathrm{eV}$ at $T_{H}^{h}=T_{H}=800 \mathrm{~K}$. The electrochemical potentials are not equal, even if the carriers are supposed to be in an isothermal regime at $T_{H}^{e}=T_{H}^{h}=T_{H}=800 \mathrm{~K}$.

Hence, all 4 thermodynamic parameters describing the two carrier populations can actually be determined from luminescence experiment as a function of a single parameter: the temperature of one of the two carriers. It is not possible to determine a priori its value, as the carrier temperatures $T_{H}^{e}, T_{H}^{h}$ are related to the relaxation dynamics of the system and depend on the asymmetry of the thermalization rates of electrons and holes.

\subsection{Implications in hot carrier selective contacts design}

Hot carrier solar cells involve carrier distribution in thermal non-equilibrium [21]. In order to take advantage of the hot carrier effect, the excess of kinetic energy of the carriers has to be converted into electrical energy in energy selective contacts [46]. Whereas some experimental works show suitable structures for hot carrier energy selective contacts [47-49], the role of each contact is not yet clear, mainly because of the different assumptions about the temperature of the carriers.

Indeed, as long as the effective mass parameter $\alpha$ is lower than 0.05 in the studied materials, the hot carrier effect can be seen even if only the energy selective contact for electrons is designed. The emission temperature measured by the generalized Planck's law $\left(T_{H}\right.$ in expression (1)) slightly depends on the hole temperature.

For $0.95<\alpha<1$, only one energy selective contact for holes needs to be designed, because the emission temperature is mainly due to the holes and the temperature of the electrons is unknown.

For $0.05<\alpha<0.95$ one type of carrier may be hotter than the emission temperature and the other colder. So two types of energy selective contacts are needed.

Moreover, the considerations about the symmetry of the absolute electrochemical potentials show that they are highly dependant on the temperature of the carrier. That is, the energy selective contacts of the hot carrier solar cell has to be designed accordingly.

\section{Conclusion}

Planck's radiation law has been previously generalized to semiconducting light emitting materials, which behave differently from pure black-bodies. This approach was focused on an isothermal case implying a thermal equilibrium between the carriers, the lattice and the radiation. With this work we have the clear relationship between the emission temperature and the FermiDirac distribution temperature of each carrier type.

Remarkably, the generalized Planck's law still yields a single radiation temperature and radiation chemical potential, even though the material system is actually defined by three temperatures (electrons, holes and lattice) and two chemical potentials. Boundary values are given for each of these parameters. Moreover, if the effective mass and the emission temperature are known, we show that estimates of temperatures and electrochemical potentials can be obtained from photoluminescence spectra.

\section{Acknowledgments}

The authors are grateful to the French ministry of Ecology, Sustainable Development and Energy for the PhD grant of François Gibelli and to NextPV and the French embassy in Japan for a visit of François Gibelli there, where some of the work could be carried out. Part of this work was performed in the framework of the program "New concepts in photovotaics" at IPVF under contract ANR-IPVF-02.

\section{Appendix A. Generalized Planck's law for different parabolic band structures}

\section{A.1. Method}

Whatever the dispersion relation, it is possible to write a 
generalized Planck's law with different electron and hole temperatures starting from expression (2), and writing:

- the k-dependant energy of the electron $E^{e}$ and of the hole $E^{h}$ relative to a reference energy $E_{\text {ref }}$ and with known parameters (effective masses, constants, confinement energies, etc.);

- from $E^{e}-E^{h}=\hbar \omega$, the expression of $\mathbf{k}$ as a function of $\hbar \omega$;

- substituting $\mathbf{k}$ in the energies of the electron $E^{e}$ and of the hole $E^{h}$ with the $\hbar \omega$ expression found at the previous step;

- replacing $E^{e}$ and $E^{h}$ with these new expressions in relation (2).

\section{A.2. Quantum well}

For the quantum well structure, the dispersion relationship is considered only between the $i$ th and the $j$ th levels and their respective confinement energies:

$E^{e, i}=\frac{E_{G}}{2}+E_{c}^{i}+\frac{\hbar^{2} \mathbf{k}_{\| l}^{2}}{2 m^{e}} E^{h, j}=-\frac{E_{G}}{2}-\left|E_{v}^{j}\right|-\frac{\hbar^{2} \mathbf{k}_{\|}^{2}}{2 m^{h}}$

from this dispersion relationship:

$\mathbf{k}_{\| l}^{2}=\frac{2}{\hbar^{2}}\left(\hbar \omega-E_{G}-E_{c}^{i}-\left|E_{v}^{j}\right|\right)\left(\frac{m^{e} m^{h}}{m^{e}+m^{h}}\right)$

substituting $\mathbf{k}_{/ /}$in the electron and hole energies above:

$$
\begin{aligned}
E^{e, i}= & \frac{E_{G}}{2}+E_{c}^{i}+\left(\hbar \omega-E_{G}-E_{c}^{i}-\left|E_{v}^{j}\right|\right)\left(\frac{m^{h}}{m^{e}+m^{h}}\right) E^{h, j}=-\frac{E_{G}}{2}-\mid E_{v}^{j} \\
& ।-\left(\hbar \omega-E_{G}-E_{c}^{i}-\left|E_{v}^{j}\right|\right)\left(\frac{m^{e}}{m^{e}+m^{h}}\right)
\end{aligned}
$$

With these expressions, the generalized Planck's law for a quantum well structure is given by the expression (4):

$$
\begin{aligned}
I_{P L, Q W e}(\hbar \omega)= & C\left[\operatorname { e x p } \left(\frac { 1 } { k _ { B } } ( \frac { 1 } { T _ { H } ^ { e } } - \frac { 1 } { T _ { H } ^ { h } } ) \left[\left(\frac{m^{e}}{m^{e}+m^{h}}\right)\left(\frac{E_{G}}{2}+E_{c}^{i}\right)\right.\right.\right. \\
& \left.-\left(\frac{m^{h}}{m^{e}+m^{h}}\right)\left(\frac{E_{G}}{2}+\mid E_{v}^{j_{l}}\right)\right]+\frac{\hbar \omega}{k_{B}\left(m^{e}+m^{h}\right)}\left(\frac{m^{h}}{T_{H}^{e}}+\frac{m^{e}}{T_{H}^{h}}\right) \\
& \left.\left.-\frac{\mu_{H}^{e}}{k_{B} T_{H}^{e}}-\frac{\mu_{H}^{h}}{k_{B} T_{H}^{h}}\right)-1\right]^{-1}
\end{aligned}
$$

\section{A.3. Quantum wire}

In a quantum wire, the energy of the electron in the $i$ th conduction subband at energy $E_{c}^{i}$ and of the hole in the $j$ th valence subband at energy $E_{v}^{j}$ which are involved in a radiative inter subband transition are given with the respective confinement energies:

$E^{e, i}=\frac{E_{G}}{2}+E_{c, y}^{i}+E_{c, z}^{i}+\frac{\hbar^{2} \mathbf{k}_{x}^{2}}{2 m^{e}} E^{h, j}=-\frac{E_{G}}{2}-\left|E_{v, y}^{j}\right|-\left|E_{v, z}^{j}\right|-\frac{\hbar^{2} \mathbf{k}_{x}^{2}}{2 m^{h}}$

from this dispersion relationship:

$\mathbf{k}_{x}^{2}=\frac{2}{\hbar^{2}}\left(\frac{m^{e} m^{h}}{m^{e}+m^{h}}\right)\left(\hbar \omega-E_{G}-E_{c, y}^{i}-\left|E_{v, y}^{j}\right|-E_{c, z}^{i}-\left|E_{v, z}^{j}\right|\right)$

substituting $\mathbf{k}_{x}$ in the electron and hole energies above:

$$
\begin{aligned}
E^{e, i}= & \frac{E_{G}}{2}+E_{c, y}^{i}+E_{c, z}^{i}+\left(\frac{m^{h}}{m^{e}+m^{h}}\right)\left(\hbar \omega-E_{G}-E_{c, y}^{i}-\left|E_{v, y}^{j}\right|-E_{c, z}^{i}-\right. \\
& \left.\left|E_{v, z}^{j}\right|\right) E^{h, j}=-\frac{E_{G}}{2}-\left|E_{v, y}^{j}\right|-\left|E_{v, z}^{j}\right|-\left(\frac{m^{e}}{m^{e}+m^{h}}\right)\left(\hbar \omega-E_{G}-E_{c, y}^{i}-\right. \\
& \left.\left|E_{v, y}^{j}\right|-E_{c, z}^{i}-\left|E_{v, z}^{j}\right|\right)
\end{aligned}
$$

With these expressions, the generalized Planck's law for a quantum wire writes:

$$
\begin{aligned}
I_{P L, Q W i}(\hbar \omega)=C(\hbar \omega) & {\left[\operatorname { e x p } \left(\frac { 1 } { k _ { B } } ( \frac { 1 } { T _ { H } ^ { e } } - \frac { 1 } { T _ { H } ^ { h } } ) \left[\left(\frac{m^{e}}{m^{e}+m^{h}}\right)\left(\frac{E_{G}}{2}+E_{c, y}^{i}+E_{c, z}^{i}\right)\right.\right.\right.} \\
& \left.-\left(\frac{m^{h}}{m^{e}+m^{h}}\right)\left(\frac{E_{G}}{2}+\left|E_{v, y}^{j}\right|+\left|E_{v, z}^{j}\right|\right)\right] \\
& \left.\left.+\frac{\hbar \omega}{k_{B}\left(m^{e}+m^{h}\right)}\left(\frac{m^{h}}{T_{H}^{e}}+\frac{m^{e}}{T_{H}^{h}}\right)-\frac{\mu_{H}^{e}}{k_{B} T_{H}^{e}}-\frac{\mu_{H}^{h}}{k_{B} T_{H}^{h}}\right)-1\right]^{-1}
\end{aligned}
$$

$C=A(\hbar \omega) \frac{\Omega n_{o p}^{2}(\hbar \omega)^{2}}{4 \pi^{2} \hbar^{3} c^{2}}$

For quantum well and quantum wire, only the fundamental transitions in the confinement directions have been considered; the reader has to be aware that for other transitions the absorption $A(\hbar \omega)$ is highly modified.

\section{Appendix B. Carrier temperatures and effective mass ratio relationship}

The emission temperature is given from the generalized Planck's law with two temperatures as:

$\frac{1}{T_{H}}=\frac{1}{m^{e}+m^{h}}\left(\frac{m^{e}}{T_{H}^{h}}+\frac{m^{h}}{T_{H}^{e}}\right)$

multiplying by $T_{H}$ :

$1=\frac{m^{e}}{m^{e}+m^{h}} \frac{T_{H}}{T_{H}^{h}}+\frac{m^{h}}{m^{e}+m^{h}} \frac{T_{H}}{T_{H}^{e}}$

introducing $\alpha=m^{e} /\left(m^{e}+m^{h}\right)$ :

$$
\begin{aligned}
1= & \alpha \frac{T_{H}}{T_{H}^{h}}+(1-\alpha) \frac{T_{H}}{T_{H}^{e}} \\
& \left.\alpha=m^{e} /\left(m^{e}+m^{h}\right) \in\right] 0,1[.
\end{aligned}
$$

\section{Appendix C. Electroneutrality and $\Delta \mu_{H}$}

For a 3D density of states under parabolic band approximation, the electroneutrality is given by:

$$
\left(\frac{m^{e} T_{H}^{e}}{m^{h} T_{H}^{h}}\right)^{3 / 2}=\exp \left(\frac{E_{G}}{2 k_{B}}\left(\frac{1}{T_{H}^{e}}-\frac{1}{T_{H}^{h}}\right)+\frac{\mu_{H}^{h}}{k_{B} T_{H}^{h}}-\frac{\mu_{H}^{e}}{k_{B} T_{H}^{h}}\right)
$$

that is, for the electrons:

$$
-\mu_{H}^{e}=k_{B} T_{H}^{e} \ln \left[\left(\frac{m^{e} T_{H}^{e}}{m^{h} T_{H}^{h}}\right)^{3 / 2} \exp \left(-\frac{E_{G}}{2 k_{B}}\left(\frac{1}{T_{H}^{e}}-\frac{1}{T_{H}^{h}}\right)-\frac{\mu_{H}^{h}}{k_{B} T_{H}^{h}}\right)\right]
$$

this last expression can be introduced in the expression (9) giving $\Delta \mu_{H}$ :

$\frac{\mu_{H}^{h}}{T_{H}^{h}}=\frac{\Delta \mu_{H}}{T_{H}}+\frac{E_{G}}{2}\left(\frac{m^{e}-m^{h}}{m^{e}+m^{h}}\right)\left(\frac{1}{T_{H}^{e}}-\frac{1}{T_{H}^{h}}\right)-\frac{\mu_{H}^{e}}{T_{H}^{e}}$

to give: 


$$
\begin{aligned}
\mu_{H}^{h}= & \frac{T_{H}^{h}}{2}\left[\frac{\Delta \mu_{H}}{T_{H}}+\frac{E_{G}}{2}\left(\frac{m^{e}-m^{h}}{m^{e}+m^{h}}\right)\left(\frac{1}{T_{H}^{e}}-\frac{1}{T_{H}^{h}}\right)+\frac{3}{2} k_{B} \ln \left(\frac{m^{e} T_{H}^{e}}{m^{h} T_{H}^{h}}\right)\right. \\
& \left.-\frac{E_{G}}{2}\left(\frac{1}{T_{H}^{e}}-\frac{1}{T_{H}^{h}}\right)\right]
\end{aligned}
$$

Doing the same work on the holes and substituting the effective masses by $\alpha$ lead to the expressions given in the section about the electrochemical potentials.

\section{References}

[1] Max Planck, Vorlesungen über die Theorie der Warmestrahlung, Verlag von Johann-Ambrosius Barth, Leipzig, 1906.

[2] Gordon Lasher, Frank Stern, Spontaneous and stimulated recombination radiation in semiconductors, Phys. Rev. 133 (January (2A)) (1964) A553-A563.

[3] A. De Vos, H. Pauwels, On the thermodynamic limit of photovoltaic energy conversion, Appl. Phys. 25 (2) (1981) 119-125.

[4] P. Würfel, The chemical potential of radiation, J. Phys. C: Solid State Phys. 15 (18) (1982) 3967.

[5] E. Daub, P. Würfel, Ultralow values of the absorption coefficient of Si obtained from luminescence, Phys. Rev. Lett. 74 (February (6)) (1995) 1020-1023.

[6] Frank Stern, Dispersion of the index of refraction near the absorption edge of semiconductors, Phys. Rev. 133 (March (6A)) (1964) A1653-A1664.

[7] P.T. Landsberg, Electron interaction effects on recombination spectra, Phys. Status Solidi B 15 (2) (1966) 623-626.

[8] B. Feuerbacher, P. Würfel, Verification of a generalised Planck law by investigation of the emission from GaAs luminescent diodes, J. Phys.: Condens. Matter 2 (April (16)) (1990) 3803-3810.

[9] T. Trupke, R.A. Bardos, M.D. Abbott, Self-consistent calibration of photoluminescence and photoconductance lifetime measurements, Appl. Phys. Lett. 87 (18) (2005) 184102.

[10] Y. Rosenwaks, M.C. Hanna, D.H. Levi, D.M. Szmyd, R.K. Ahrenkiel, A.J. Nozik, Hot-carrier cooling in GaAs: quantum wells versus bulk, Phys. Rev. B 48 (November (19)) (1993) 14675-14678.

[11] Paul Berdahl, Radiant refrigeration by semiconductor diodes, J. Appl. Phys. 58 (3) (1985) 1369.

[12] Arthur J. Nozik, Spectroscopy and hot electron relaxation dynamics in semiconductor quantum wells and quantum dots, Ann. Rev. Phys. Chem. 52 (October (1)) (2001) 193-231.

[13] P. Würfel, Solar energy conversion with hot electrons from impact ionisation, Sol. Energy Mater. Sol. Cells 46 (1997) 43-52.

[14] P.T. Landsberg, H.S. Leff, Thermodynamic cycles with nearly universal maximum-work efficiencies, J. Phys. A: Math. Gen. 22 (September (18)) (1989) 4019-4026.

[15] G. Smestad, H. Ries, Luminescence and current-voltage characteristics of solar cells and optoelectronic devices, Sol. Energy Mater. Sol. Cells 25 (January (12)) (1992) 51-71.

[16] Amaury Delamarre, Laurent Lombez, Jean-François Guillemoles, Contactless mapping of saturation currents of solar cells by photoluminescence, Appl. Phys. Lett. 100 (13) (2012) 131108.

[17] K. Schick, E. Daub, S. Finkbeiner, P. Würfel, Verification of a generalized Planck law for luminescence radiation from silicon solar cells, Appl. Phys. A Solids Surf. 54 (February (2)) (1992) 109-114.

[18] David Regesch, Levent Gütay, Jes K. Larsen, Valérie Deprédurand, Daisuke Tanaka, Yasuhiro Aida, Susanne Siebentritt, Degradation and passivation of CuInSe[sub 2], Appl. Phys. Lett. 101 (11) (2012) 112108.

[19] E. Takeda, A. Shimizu, T. Hagiwara, Role of hot-hole injection in hot-carrie effects and the small degraded channel region in MOSFET's, IEEE Electron Device Lett. 4 (September (9)) (1983) 329-331.

[20] K.R. Hofmann, C. Werner, W. Weber, G. Dorda, Hot-electron and hole-emission effects in short n channel MOSFET's, IEEE Trans. Electron Devices 32 (March (3)) (1985) 691-699.

[21] Robert T. Ross, Efficiency of hot-carrier solar energy converters, J. Appl. Phys 53 (5) (1982) 3813.

[22] F. Binet, J.Y. Duboz, J. Off, F. Scholz, High-excitation photoluminescence in GaN: hot-carrier effects and the Mott transition, Phys. Rev. B 60 (August (7)) (1999) 4715-4722.

[23] Marcus Freitag, Vasili Perebeinos, Jia Chen, Aaron Stein, James C. Tsang, James A. Misewich, Richard Martel, Phaedon Avouris, Hot carrier electroluminescence from a single carbon nanotube, Nano Lett. 4 (June (6)) (2004) 1063-1066.
[24] Dong Sun, Grant Aivazian, Aaron M. Jones, Jason S. Ross, Wang Yao, David Cobden, Xiaodong Xu, Ultrafast hot-carrier-dominated photocurrent in graphene, Nat. Nanotechnol. 7 (January (2)) (2012) 114-118.

[25] N.M. Gabor, J.C.W. Song, Q. Ma, N.L. Nair, T. Taychatanapat, K. Watanabe, T. Taniguchi, L.S. Levitov, P. Jarillo-Herrero, Hot carrier-assisted intrinsic photoresponse in graphene, Science 334 (November (6056)) (2011) 648-652.

[26] Jens Christian Johannsen, Sren Ulstrup, Federico Cilento, Alberto Crepaldi, Michele Zacchigna, Cephise Cacho, I.C. Turcu, Emma Springate, Felix Fromm, Christian Raidel, et al., Direct View on the Ultrafast Carrier Dynamics in Graphene, arXiv preprint arXiv:1304.2615, 2013.

[27] Qi Chen, Chunfeng Zhang, Fei Xue, Yong Zhou, Wei Li, Ye Wang, Wenguang Tu, Zhigang Zou, Xiaoyong Wang, Min Xiao, Enhanced hot-carrier luminescence in multilayer reduced graphene oxide nanospheres, Sci. Rep. 3 (July) (2013).

[28] R.S. Sundaram, M. Engel, A. Lombardo, R. Krupke, A.C. Ferrari, Ph. Avouris, M. Steiner, Electroluminescence in single layer $\mathrm{MoS}_{2}$, Nano Lett. 13 (April (4)) (2013) 1416-1421.

[29] S.A. Lyon, Spectroscopy of hot carriers in semiconductors, J. Lumin. 35 (June (3)) (1986) 121-154.

[30] A. Twardowski, C. Hermann, Polarized hot-electron photoluminescence in highly doped GaAs, Phys. Rev. B 32 (December (12)) (1985) 8253-8257.

[31] Louise C. Hirst, Hiromasa Fujii, Yunpeng Wang, Masakazu Sugiyama, Nicholas J. Ekins-Daukes, Hot carriers in quantum wells for photovoltaic efficiency enhancement, IEEE J. Photovolt. 4 (January (1)) (2014) 244-252.

[32] A. Le Bris, L. Lombez, S. Laribi, G. Boissier, P. Christol, J.-F. Guillemoles, Thermalisation rate study of GaSb-based heterostructures by continuous wave photoluminescence and their potential as hot carrier solar cell absorbers, Energy Environ. Sci. 5 (3) (2012) 6225.

[33] Jean Rodière, Laurent Lombez, Alain Le Corre, Olivier Durand, JeanFrançois Guillemoles, Experimental evidence of hot carriers solar cell operation in multi-quantum wells heterostructures, Appl. Phys. Lett. 106 (May (18)) (2015) 183901.

[34] J. Bude, K. Hess, G.J. Iafrate, Impact ionization in semiconductors: effects of high electric fields and high scattering rates, Phys. Rev. B 45 (May (19)) (1992) 10958-10964.

[35] N. Tsutsu, Y. Uraoka, Y. Nakata, S. Akiyama, H. Esaki, New Detection Method of Hot-Carrier Degradation Using Photon Spectrum Analysis of Weak Luminescence on CMOS VLSI, IEEE, 1990, pp. 143-148.

[36] Yasuhiko Takeda, Tadashi Ito, Ryo Suzuki, Tomoyoshi Motohiro, Santosh Shrestha, Gavin Conibeer, Impact ionization and Auger recombination at high carrier temperature, Sol. Energy Mater. Sol. Cells 93 (June (6-7)) (2009) 797-802.

[37] P. Aliberti, Y. Feng, Y. Takeda, S.K. Shrestha, M.A. Green, G. Conibeer, Investigation of theoretical efficiency limit of hot carriers solar cells with a bulk indium nitride absorber, J. Appl. Phys. 108 (9) (2010) 094507.

[38] P. Würfel, A.S. Brown, T.E. Humphrey, M.A. Green, Particle conservation in the hot-carrier solar cell, Prog. Photovolt.: Res. Appl. 13 (June (4)) (2005) 277-285.

[39] Antonio Luque, Antonio Martí, Electron phonon energy transfer in hot-carrier solar cells, Sol. Energy Mater. Sol. Cells 94 (February (2)) (2010) 287-296.

[40] J. Shah, C. Lin, R.F. Leheny, A.E. DiGiovanni, Pump wavelength dependance of hot electron temperature in GaAs, Solid State Commun. 18 (1976) 487-489.

[41] P. Würfel (Ed.), Physics of Solar Cells, Wiley-VCH Verlag GmbH, Weinheim, Germany, 1997.

[42] Krzysztof Józwikowska, Alina Józwikowska, Michał Nietopiel, The non-equilibrium statistical distribution function for electrons and holes in semiconductor heterostructures in steady-state conditions, Entropy 17 (June (6)) (2015) 4110-4133.

[43] Gavin Conibeer, Nicholas Ekins-Daukes, Jean-François Guillemoles, Dirk Knig, Eun-Chel Cho, Chu-Wei Jiang, Santosh Shrestha, Martin Green, Progress on hot carrier cells, Sol. Energy Mater. Sol. Cells 93 (June (6-7)) (2009) 713-719.

[44] Andreas Othonos, H.M. van Driel, Jeff F. Young, Paul J. Kelly, Correlation of hotphonon and hot-carrier kinetics in Ge on a picosecond time scale, Phys. Rev. B 43 (March (8)) (1991) 6682-6690.

[45] Sergey L. Rumyantsev, Michael Shur, M.E. Levinshten, Handbook Series on Semiconductor Parameters, vol. 2, World Scientific, Singapore, London, 1999.

[46] Gavin Conibeer, Chu-Wei Jiang, Martin Green, Nils Harde, Axel Straub, Selective energy contacts for potential application to hot carrier PV cells, in: Proceedings of 3rd World Conference on Photovoltaic Energy Conversion, 2003, vol. 3, IEEE, 2003, pp. 2730-2733.

[47] G.J. Conibeer, C.-W. Jiang, D. König, S. Shrestha, T. Walsh, M.A. Green, Selective energy contacts for hot carrier solar cells, Thin Solid Films 516 (August (20)) (2008) 6968-6973.

[48] P. Aliberti, S.K. Shrestha, R. Teuscher, B. Zhang, M.A. Green, G.J. Conibeer, Study of silicon quantum dots in a $\mathrm{SiO}_{2}$ matrix for energy selective contacts applications, Sol. Energy Mater. Sol. Cells 94 (November (11)) (2010) 1936-1941.

[49] James A.R. Dimmock, Stephen Day, Matthias Kauer, Katherine Smith, Jon Heffernan, Demonstration of a hot-carrier photovoltaic cell, Prog. Photovolt.: Res. Appl. 22 (February (2)) (2014) 151-160. 